\title{
Induction thermography for automatic crack detection in automotive components
}

\author{
by L. Franco*, F. Rodríguez* and J. Otero*
}

\begin{abstract}
*AIMEN Technology Center, C/Relva 27A O Porriño, Pontevedra, Spain, Ifranco@aimen.es,
\end{abstract} francisco.rodriguez@aimen.es

\begin{abstract}
Active thermography with inductive excitation is proposed as a reliable method for substitution of magnetic particle inspection on steel automotive components. A study for inner part inspection of the component is presented, where typical superficial cracks of different length $(>5 \mathrm{~mm})$ and orientation are detected by induction thermography. Programmed acquisition and analysis with Fourier transformation are developed to obtain a phase image for crack detection. Image processing can be developed for a reliable detection of the crack within the geometrical indications. Short induction and testing times per view will allow acceptable inspection times for the complete steel parts.
\end{abstract}

\section{Introduction}

Infrared thermography has been probed as a successful NDT\&E technique in many applications and materials. It can be applied to both metals and non-metals (as its extended applications in composite materials for aeronautics) containing subsurface defects [1]. In particular, active thermography is based on applying an external source of energy to induce a temperature difference between defective and non-defective areas in a probe under inspection. This difference is consequently imaged by a thermal camera, and later image processing can be developed in the acquired sequence of thermal images. Defect detection within these images can be resolved by several methods, as for example direct visualization, Fourier processing or Thermographic Signal Reconstruction [2].

Induction (or induction heating) thermography is based on the induction of Eddy currents in the inspected probe as the source of energy. Basically, an induction coil with a short electric pulse (typically from $50 \mathrm{~ms}$ to $1 \mathrm{~s}$ ) is applied (without contact) to the object of interest, thus generating Eddy currents in the surface. The Eddy currents' distribution varies when a defect is present, generating a heat profile in the defect which can be imaged with a thermal camera [3],[4]. This technique is suitable for inspecting electrically conductive materials and also for CFRP. Nevertheless its primary interest is for ferromagnetic steel, where subsurface defects can be easily detected. In this work we present a detailed study for detection of superficial cracks in the inner part of steel automotive components.

\subsection{Induction thermography}

Induction thermography is a non-contact ND method which, compared to traditional and successful methods, as magnetic particles, can be automated for reliable solutions.

In general, inner steel components in industry present surface and subsurface cracks which are originated on the cooling process after the process heating during its fabrication. The quality control for these defects is actually based on magnetic particle inspection, and it is applied to every single piece. The complex geometry of the components and the superficial nature of cracks avoid other traditional methods, and the evaluation must be manually performed by experienced operators.

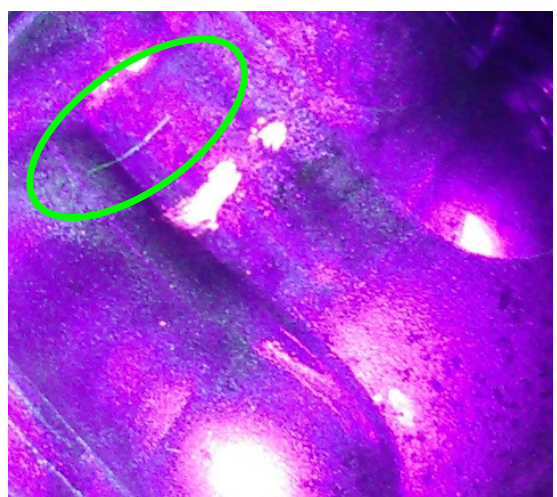

Fig. 1: Steel part under magnetic particle inspection. The crack is indicated with a green marker. 


\subsection{1/qirt.2016.165}

A high frequency induction coil in the neighbourhood of the piece generates Eddy currents in the surface. Crack detection is based on the change of the distribution of the induced Eddy currents due to the crack. These changes lead to local Eddy current loses near the crack, generating a heat increase that can be imaged with a thermal camera. Further heating in the piece due to the induction effect can mask the initial thermal difference on the crack.

The orientation of the Eddy currents is crucial for a reliable detection, with a maximum temperature difference for perpendicular-placed cracks. Parallel or tilted crack detection will depend upon the density of the induced Eddy currents (see Fig. 2).

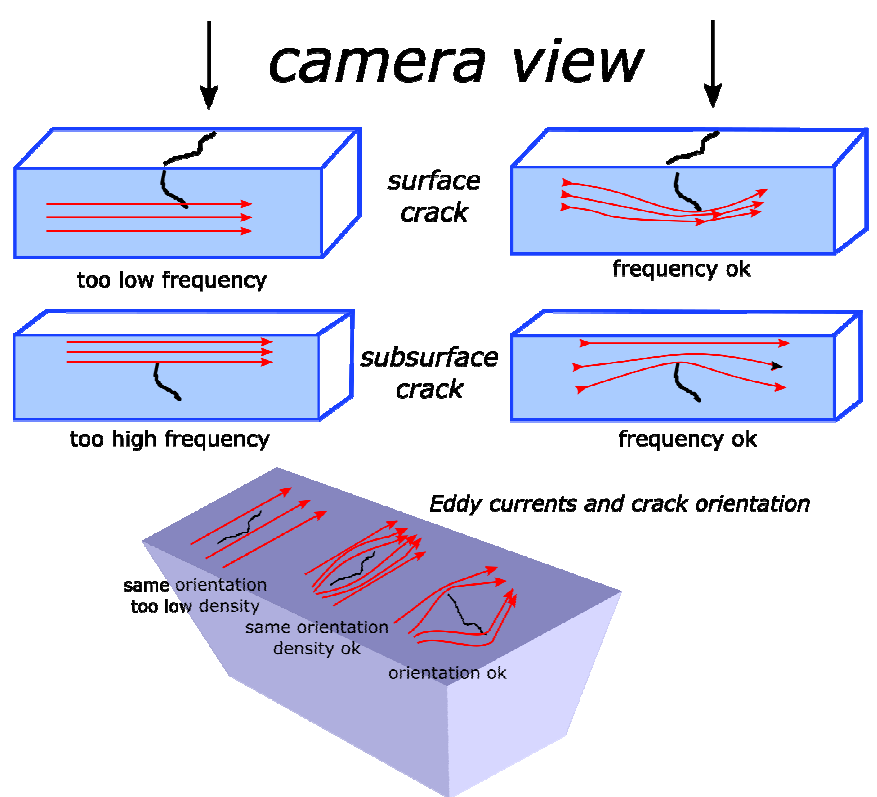

Fig. 2: Eddy currents suitable parameters for crack detection: frequency, orientation, density.

The ability to detect defects in materials by induction thermography is defined by the called characteristic lengths: electrical skin depth $\delta$ (defined by the electrical conductivity $\sigma$ and the magnetic permeability $\mu$ ) and the thermal penetration length $\left(\mu_{T}\right)$ for the material [4],[5],[6]. The frequency of the Eddy currents is a determining factor since it defines the penetration depth. The induced Eddy currents exponentially decay below the surface; they penetrate only a thin depth (skin effect) for medium and high frequencies. The coefficient of this effect is the penetration depth [4], defined as the skin depth at which the current density of frequency $f$ has dropped to $1 / e$ of the current density on the surface. It can be calculated with the expression in Eq. (1).

where:

$$
\delta=\sqrt{\frac{1}{\pi f \sigma \mu_{r} \mu_{0}}}
$$

$\sigma$ is the specific conductivity

$\mu_{r}$ is the relative permeability and $\mu_{0}$ the permeability of vacuum

$f$ the frequency in $\mathrm{Hz}$.

In general, ferromagnetic materials $\left(\mu_{r}=50-80000\right)$ show a low skin depth for $100-200 \mathrm{kHz}$, which is very relevant for detecting surface cracks. Particularly for the material in the study, carbon steel, the parameters are: $\sigma=$ $6.99 \cdot 10^{6} \mathrm{~S} / \mathrm{m}, \mu_{r}=100, \mu_{0}=4 \pi \cdot 10^{-7} \mathrm{H} / \mathrm{m}$. Considering a frequency value up to $200 \mathrm{kHz}$, the penetration depth can reach $0.04 \mathrm{~mm}$. Moreover, an effective penetration depth [7] can be defined as $\delta_{\text {eff }}=3 \delta$, the penetration depth at which the induced currents have decreased to $5 \%$ and the induction effect in the material is non-existent. Therefore, the detection depth can range from 0.04 to $0.12 \mathrm{~mm}$ for carbon steel, as the considered components in this study.

In this paper we present a study to develop a consistent and automatic non-destructive testing method based on induction thermography. Relevant results for the complex inner geometry and crack orientation have been achieved. Furthermore, results for two typical thermal cameras are shown for the evaluation of the different inspection capabilities. The aim of this work is the generation of a complete induction thermographic inspection system for steel automotive components. 


\subsection{1/qirt.2016.165}

\section{Method and materials}

\subsection{Experimental set-up}

The inspection system for induction thermography was developed using an industrial induction machine (Digimac generator and MPC-1 control from GH Induction, http://www.ghinduction.com). The maximum output power is up to $10 \mathrm{~kW}$ and the frequency is up to $200 \mathrm{kHz}$, which is automatically coupled to the load.

The induction system allows the choice of the output power (as a percentage of the maximum $10 \mathrm{~kW}$ ) and the induction time, in steps of $10 \mathrm{~ms}$. A minimum induction time of $100 \mathrm{~ms}$ was established for all the experiments.

\subsubsection{Thermal cameras}

Two thermal cameras have been employed in the study: a cooled InSb FLIR SC7600 within MWIR range (3-5 $\mu \mathrm{m})$ and an uncooled microbolometer Gobi 640GigE from Xenics within LWIR $(8-14 \mu \mathrm{m})$. The employed lenses were a $25 \mathrm{~mm} \mathrm{~F} / 2$ for the FLIR camera and a $25 \mathrm{~mm} \mathrm{F/1} \mathrm{for} \mathrm{the} \mathrm{Xenics} \mathrm{one.}$

The principal differences between the two cameras are shown in Table 1.

Table 1: Imaging characteristics of the thermal cameras (vendor's data).

\begin{tabular}{|l|c|c|}
\hline Characteristics & FLIR SC7600 & Xenics Gobi 640GigE \\
\hline Framerate (at maximum frame size) & $100 \mathrm{fps}$ & $50 \mathrm{fps}$ \\
NETD & $20 \mathrm{mK}$ & $50 \mathrm{mK}$ \\
Pixel pitch time (typical equivalent & $15 \mu \mathrm{m}$ & $17 \mu \mathrm{m}$ \\
Response from [8]) & $\sim \sim \mu \mathrm{s}$ & $7-12 \mathrm{~ms}$ \\
detector, frop & $14 \mathrm{bits}$ \\
Bit depth & & \\
\hline
\end{tabular}

The most critical characteristic for crack detection in the study will be the sensitivity (NETD) [6], due to the fact that the temperature differences that Eddy currents induce in the crack are minimal. The pitch size is also an important parameter, since the crack width will be not detected with an insufficient spatial resolution. Spatial noise (also known as fixed pattern noise) has been corrected before image frame acquisition, by applying a non-uniformity correction (NUC) process [9] and therefore it won't affect the crack detection.
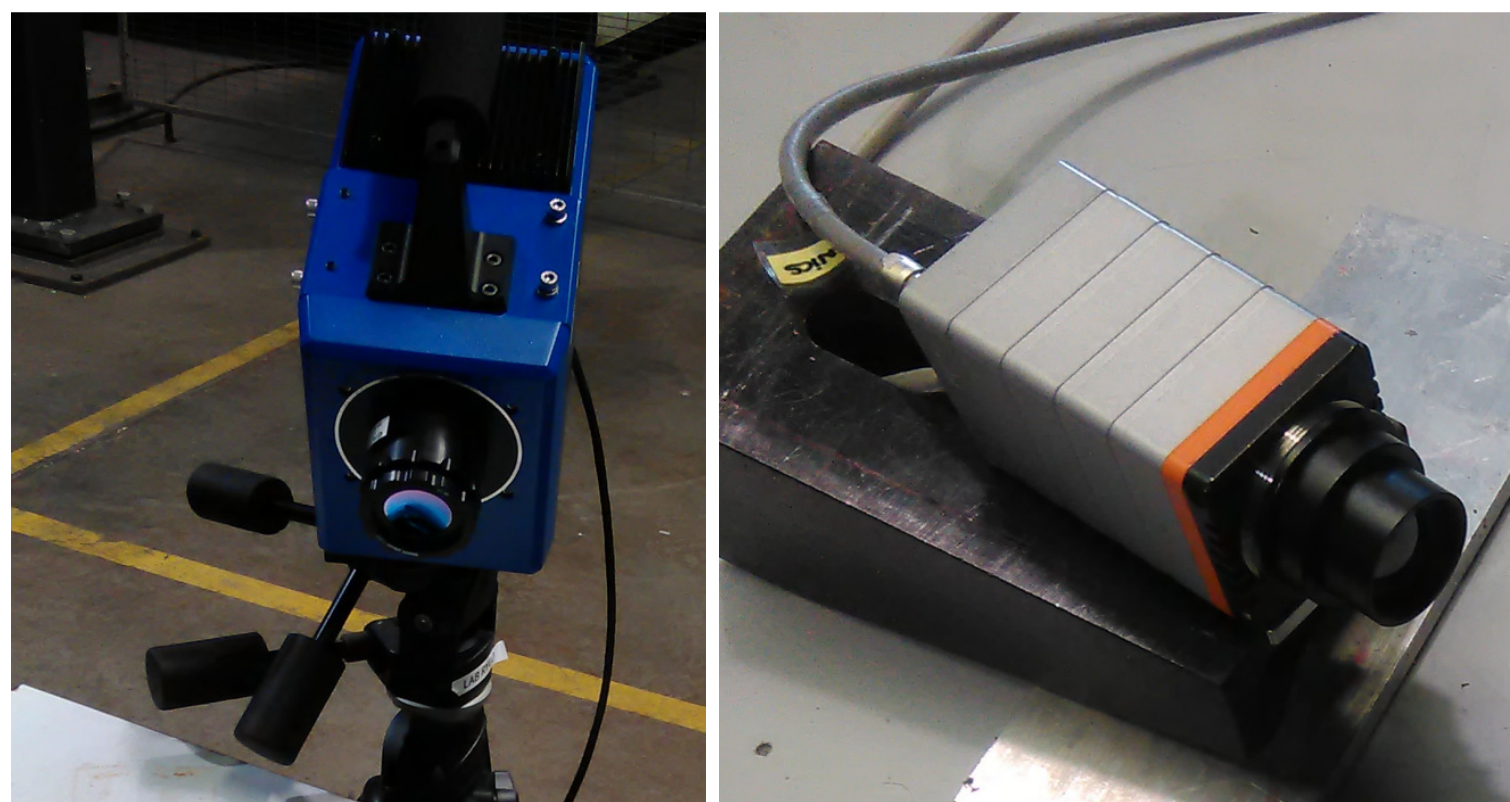

Fig. 3: Thermal cameras for the induction thermography study: FLIR SC7600 (left), Gobi 640GigE from Xenics (right). 


\subsection{1/qirt.2016.165}

\subsubsection{Acquisition and processing}

Different sequences of thermal images were analyzed and processed to obtain a base image for crack detection. The acquisition starts before the induction excitation (cold images), then the induction pulse is generated (thus the Eddy currents in the material surface) and the temperature increase in the crack is immediately seen. After the maximum temperature is generated, the heat is spread all over the affected area and the crack is no longer visualized. The induction and acquisition times must be immediate; the temperature difference in the crack is caused by the change on the Eddy currents density, not by the inducted heating. Total times for the complete sequence were fixed from 3 to 5 seconds, and a subinterval of frames of interest is lately extracted. A sequence of 64 or 128 frames is selected before the maximum temperature in the area is reached $(160-320 \mathrm{~ms})$. This interval is fixed from the temperature evolution profile of a pixel in this area (see Fig. 4).
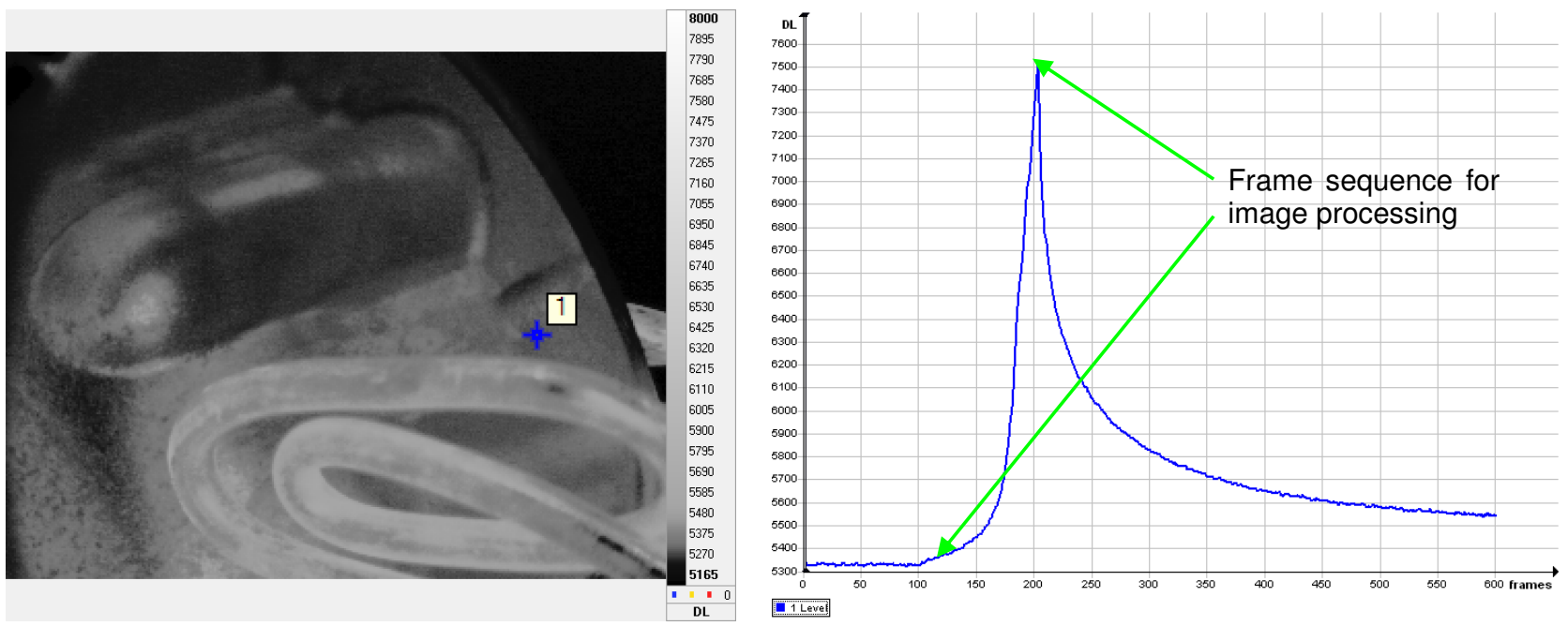

Fig. 4: Example of time evolution for a given pixel in the induction area next to the coil.

Fourier analysis is employed with this sequence, obtaining an amplitude and a phase image [10],[11]. The image sequence can be assumed as a pulsed-phase thermography where the existing differences will be detected in the frequency domain. In the induction excitation, the temperature differences arise during the heating process rather than in the consequent cooling of the piece. The phase image provides deeper probing capabilities, since it cancels part of the artifacts related to the noise in the images, coming from environmental reflections, emissivity and surface geometry variations. As a result, the crack visualization and detectability will be performed on the phase images. The crack has proved to be seen in amplitude images only for specific parameters and also depending on the thermal camera.

\subsection{Inspection samples}

Two series of carbon steel automotive pieces were inspected for this study, which present cracks in their inner part. Each series presents a different crack orientation (Fig. 5): transversal (i.e., perpendicular to the induced currents) and longitudinal (parallel to the induced currents). Transversal crack probes were named after M1 to M4, while longitudinal probes were marked as ML1 to ML4. The cracks in the steel parts are originated after the induction heating during their fabrication, and their nature depends on the part size and geometrical features. Typically, transversal cracks are originated in a change on the material structure and remain closed, while longitudinal cracks are caused by tensions between areas of different width during the heating and they can result finally in open cracks.

Series for longitudinal crack shows similar dimensions and positioning; in the transversal crack's series the indications are located on the width change except for one probe which shows a small crack in the surface (right image in Fig. 5, M2 probe). The cracks are extended over the interior elements of the components, which will result in many superficial and geometrical details that can affect the inspection and crack determination. All the cracks have larger lengths than $5 \mathrm{~mm}$. 

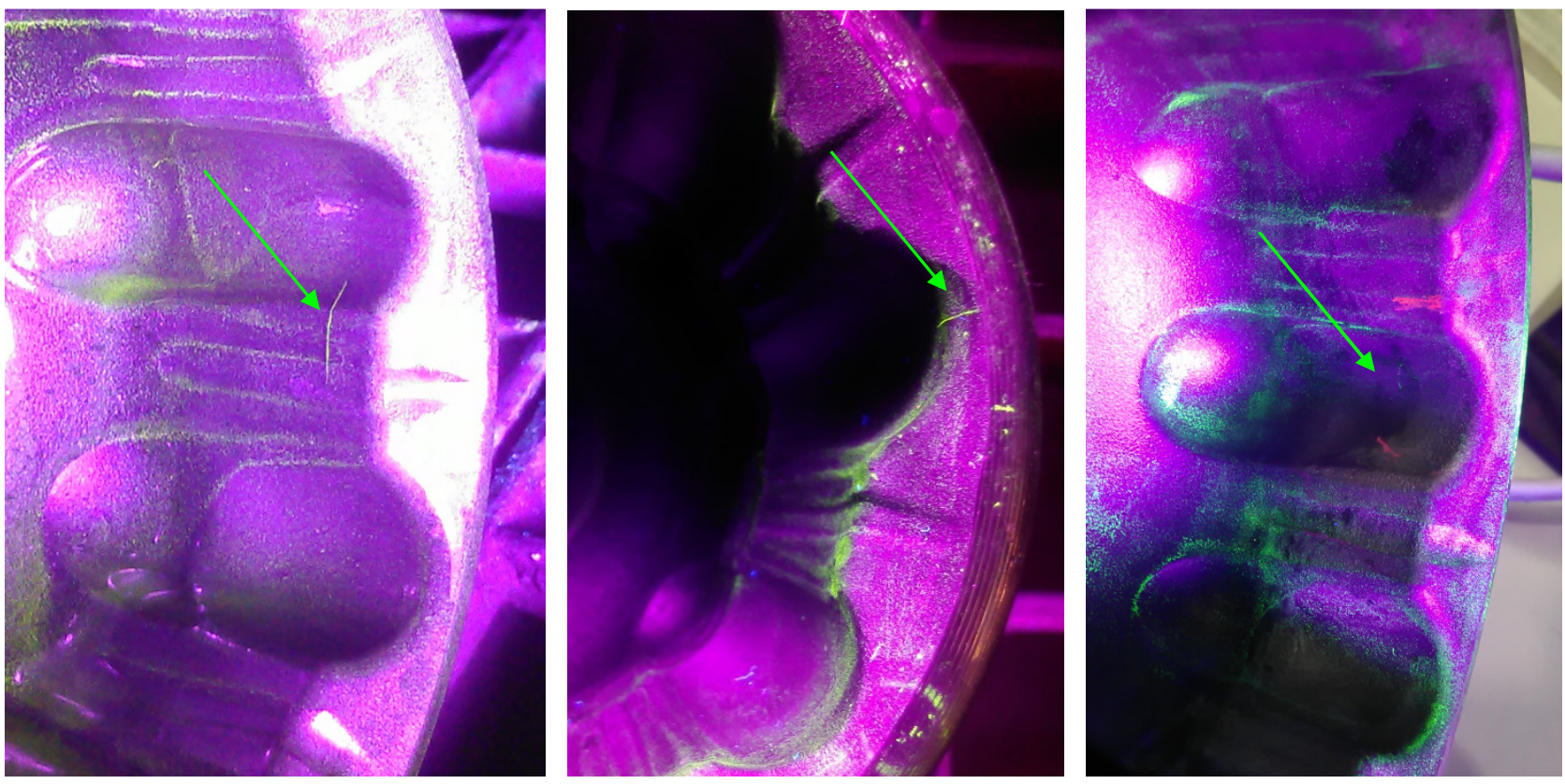

Fig. 5: Magnetic particle's photos for (left) transversal crack, (center) longitudinal crack. All the specimens show similar cracks except for (right) M2 probe, with a small transversal indication on the wall.

\subsection{Experimental parameters}

The experimental parameters for the induction excitation were chosen to optimize the crack detection and to check the detectability upon variation on the Eddy currents flux. Acquisition parameters for the detector were selected to optimize the image histogram, to obtain a reduced-noise image and a wide framerate to maximize the crack detection.

Table 2 shows the different parameters for the present study.

Table 2: Experimental parameters for induction thermography

\begin{tabular}{|c|c|c|c|}
\hline Induction parameters & $\begin{array}{r}\text { Power }(\mathrm{kW}) \\
\text { Frequency }(\mathrm{kHz}) \\
\text { Time }(\mathrm{ms}) \\
\end{array}$ & $\begin{array}{l}5,7,10 \\
150-200 \\
100 \text { to } 600(100 \mathrm{~ms} \text { steps })\end{array}$ & \\
\hline \multirow[t]{2}{*}{ Camera acquisition } & \multirow[b]{2}{*}{$\begin{array}{r}\text { Frame size (pixels) } \\
\text { Framerate (fps) } \\
\text { Integration time }(\mu s)\end{array}$} & FLIR SC7600 & Xenics Gobi 640GigE \\
\hline & & $\begin{array}{c}320 \times 256 \\
200 \\
4000\end{array}$ & $\begin{array}{c}320 \times 240 \\
100 \\
40\end{array}$ \\
\hline
\end{tabular}

Comparing the phase image for the different sequences, those with lower induction time and power show images with poorer quality (see Fig. 6 as an example). The lower quality arises from noisy initial images, where the thermal images show low contrast during the acquired sequence. 


\subsection{1/qirt.2016.165}
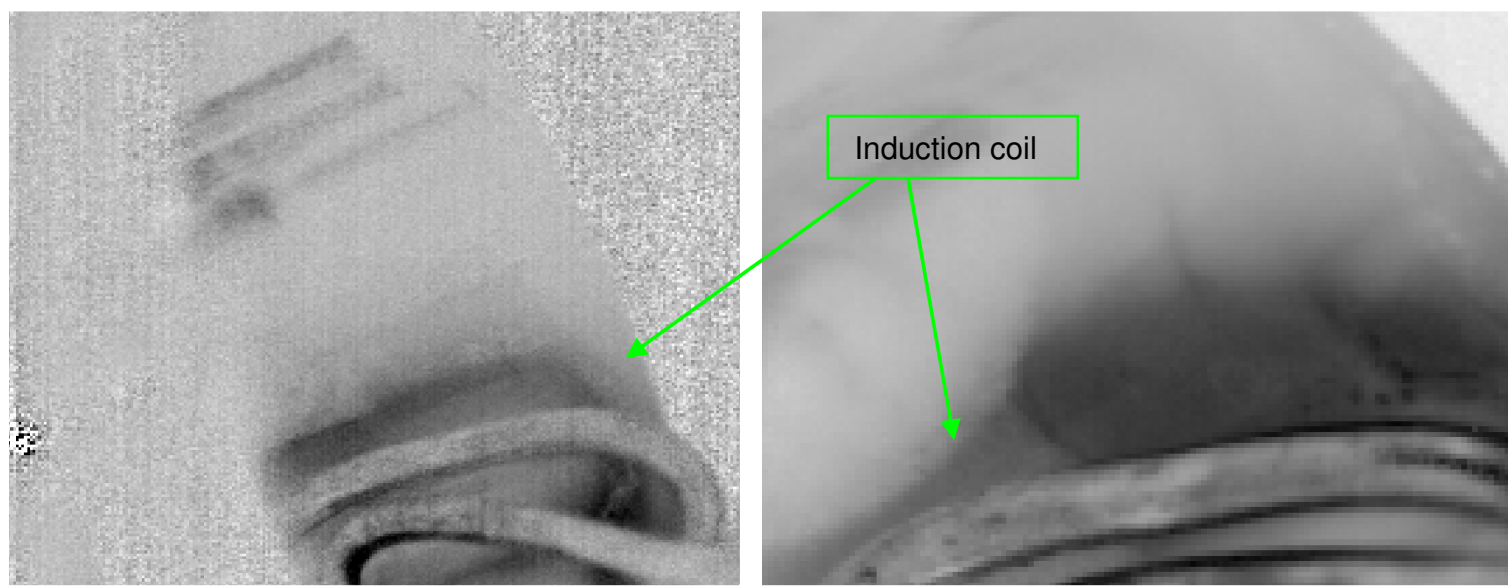

Fig. 6: Phase images: noisy (left: $5 \mathrm{~kW}, 200 \mathrm{~ms}$ ), and plain example (right: $10 \mathrm{~kW}, 500 \mathrm{~ms}$ ) for the Xenics camera.

Measurements with different coils were performed to obtain a reliable induction pattern with a simple coil positioning. This positioning will be located in the interior part for detectability, and it must be repeatable for the inspection of every piece, generating a similar current's distribution. The coils were orientated to generate Eddy currents perpendicularly to the transverse cracks. For the longitudinal cracks, additional measurements with a rectangular coil situated in the exterior part were acquired for comparison and evaluation for current's density (see Fig. 7).
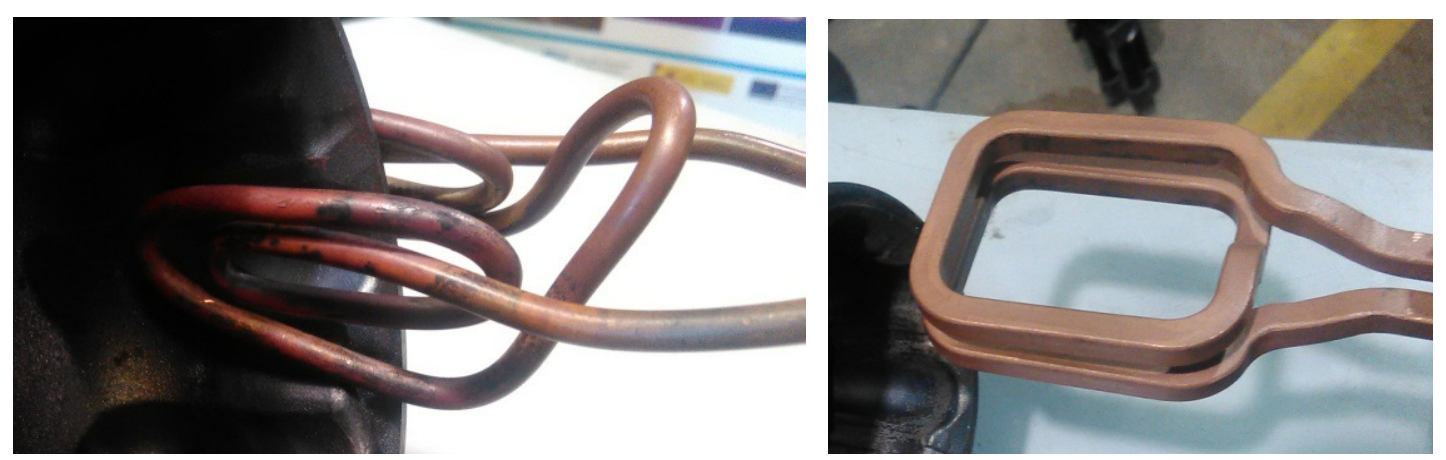

Fig. 7: Internal coil for both transversal and longitudinal (left) and external coil for longitudinal crack detection (right).

\section{Results}

The different cracks in the series (>5 mm length) are all detected except for the interior M2 part (see Fig. 5, transversal crack). The summary of the detectability and capability for both cameras and part series is shown in Table 3 . Results are referred to induction coils of Fig. 7; the rectangular coil results only refer to longitudinal cracks. Due to its size, the rectangular coil can not be located in the interior part of the pieces, therefore it was not considered for detection of transversal cracks. 


\subsection{1/qirt.2016.165}

Table 3: Detectability results for both thermal cameras.

\begin{tabular}{|c|c|c|}
\hline Part series & FLIR SC7600 & Xenics Gobi 640GigE \\
\hline $\begin{array}{l}\text { Transversal crack } \\
\text { M1, M3, M4 } \\
\text { M2 }\end{array}$ & $\begin{array}{l}\text { Visibility OK in phase, for high induction times } \\
\text { also visible in amplitude images. } \\
\text { NOT visible. }\end{array}$ & $\begin{array}{l}\text { Visibility OK in phase images for high } \\
\text { induction time values }{ }^{1} \text {. } \\
\text { NOT visible. }\end{array}$ \\
\hline $\begin{array}{l}\text { Longitudinal crack } \\
\text { ML1, ML2, ML3, ML4 } \\
\text { (internal coil) }\end{array}$ & $\begin{array}{l}\text { Visibility OK in phase images for high induction } \\
\text { times }{ }^{2} \text {. A good coil orientation improves the } \\
\text { detection. }\end{array}$ & $\begin{array}{l}\text { Visibility NOT OK, only in some cases } \\
\text { regarding the coil orientation. }\end{array}$ \\
\hline $\begin{array}{l}\text { Longitudinal crack } \\
\text { ML1, ML2, ML3, ML4 } \\
\text { (external coil) }\end{array}$ & Visibility OK for all parameters. & $\begin{array}{l}\text { Visibility OK in phase images, from } 300 \mathrm{~ms} \\
\text { induction time. }\end{array}$ \\
\hline
\end{tabular}

1 . Orientation of the coil is crucial for detection with the Xenics camera.

2. High induction times range from $500 \mathrm{~ms}$ for $5 \mathrm{~kW}$ or $400 \mathrm{~ms}$ for $7,10 \mathrm{~kW}$.
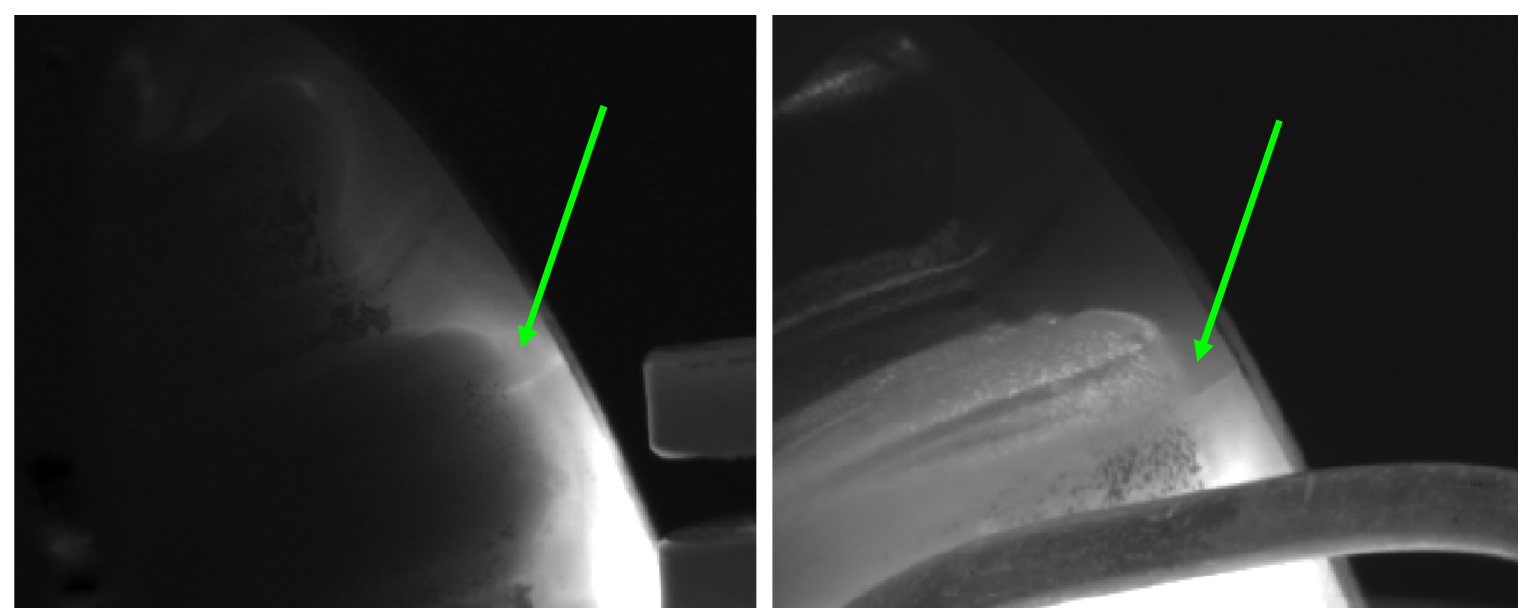

Fig. 8: Longitudinal crack for FLIR camera, (left) external coil and 400 ms, (right) internal coil 500 ms induction time.
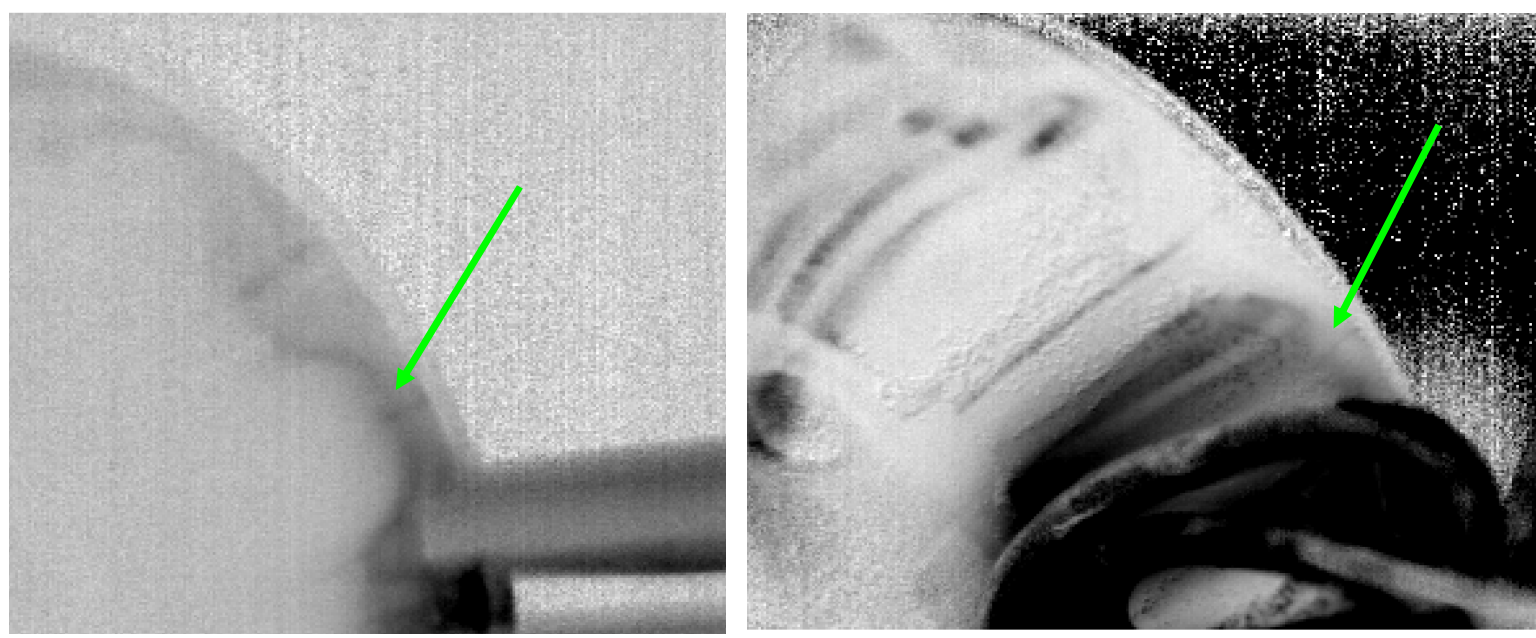

Fig. 9: Longitudinal crack for Xenics camera and 500 ms induction time, (left) external coil, (right) internal coil. 

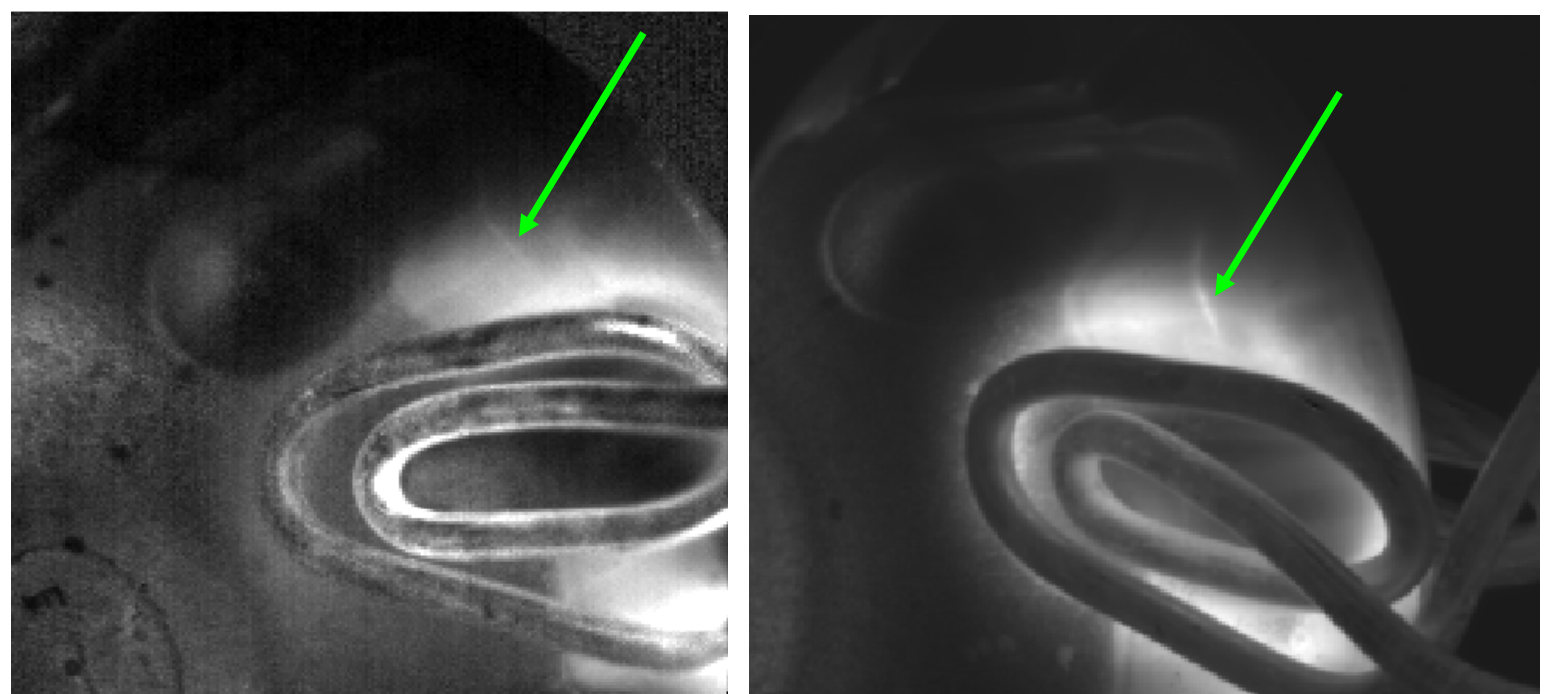

Fig. 10: Transversal cracks phase images for M1, $10 \mathrm{~kW}$ and 500 ms (left) Xenics, (right) FLIR camera.
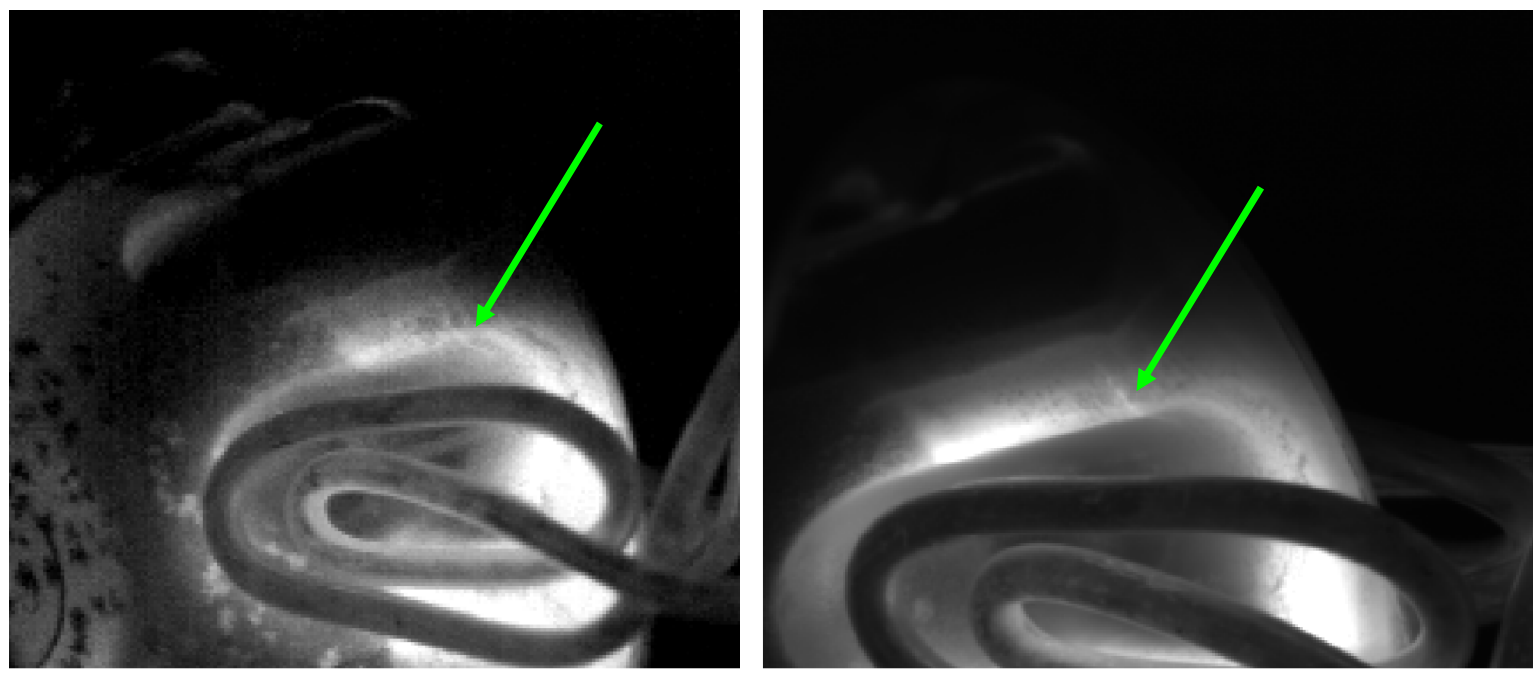

Fig. 11: Transversal crack phase images for M3, 10 kW, 400 ms (left) Xenics, (right) FLIR camera.

The coil positioning and orientation has been demonstrated as a critical parameter for crack detectability. A change in the coil positioning, as increasing the distance of the coil from the part, results in a decrease of the current density [6], which can finally be insufficient for generating the temperature difference for the crack detection.

\subsection{Analysis}

In general, the crack visualization is clear in the phase images. Nevertheless, from the point of view of a further automated detection, many different indications with an equivalent grey level to the crack's value can also be detected. These indications come from the geometrical features and other effects as the irregular surface of the piece. Moreover, the coil must be close to the crack area, to generate Eddy currents which will reach the crack for detection. Heating effect from the induced currents generate a grey level gradient in the image next to the coil, what will also affect the crack detection.

Comparing the images from both cameras, the FLIR camera generates smoother images with increased crack detectability. The dependence on the coil positioning and orientation is less critical than with the Xenics camera, where the positioning in several cases implied the non-visualization of the crack.

The crack in the M2 probe is not detected with any parameters or camera combination. In fact, it is also difficult to visualize with magnetic particles; it shows a smaller length with a less intense mark than for the other cracks. As a consequence, this part is not longer estimated for the developing of the induction thermography system. 


\subsection{1/qirt.2016.165}

A quantitative value for the crack detection depending on the acquisition parameters is of high interest. This crack detectability can be performed by comparing equivalent profiles in the images. Fig. 12 shows the crack profiles for the different acquisition parameters (power, induction time).
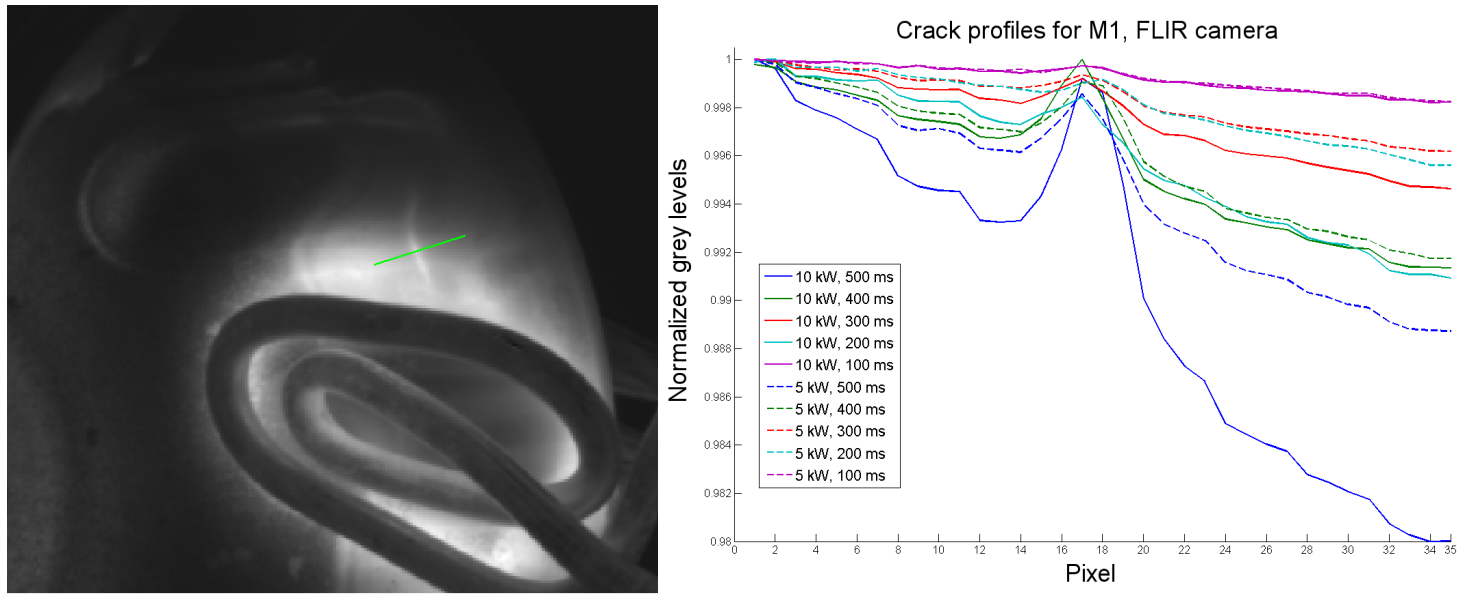

Fig. 12: Example of crack profile in M1 probe with the FLIR camera.

For these images, a quantitative comparison can be performed by calculating the normalized maximum/mean ratio for the profiles. Table 4 shows as an example the values for the transversal crack probes. In general, the crack variation over the area increases with both the power and the induction time, as expected. The indicated values are very low since they are calculated from the raw phase images, without improving the contrast or stretching the histogram.

Table 4: Quantitative variation (arbitrary units) for the crack with respect to the adjacent area

\begin{tabular}{|l|l|r|}
\hline Probe & Power $(\mathbf{k W})$ & Induction time - $\mathbf{1 0 0 , 2 0 0 , 3 0 0 , 4 0 0 , 5 0 0 ~} \mathbf{~ m s}$ \\
\hline M1 & 5 & $0.07,0.19,0.18,0.40,0.55$ \\
& 10 & $0.08,0.41,0.25,0.43,0.96$ \\
\hline M3 & 5 & $0.01,0.03,0.06,0.07,0.012$ \\
& 10 & $0.01,0.03,0.02,0.18,0.33$ \\
\hline M4 & 5 & $0.03,0.04,0.05,0.08,0.09$ \\
& 10 & $0.04,0.04,0.13,0.19,0.50$ \\
\hline
\end{tabular}

\section{Conclusions and future work}

In this work we have presented an induction thermography inspection for steel automotive parts. The study was oriented to detect interior cracks in transversal and longitudinal directions with a $>5 \mathrm{~mm}$ length in two part series of 4 elements each. This study has allowed the evaluation and validation of induction thermography for crack detection on automotive parts with a complex and specific geometry. Critical experimental conditions and parameters range values have been determined and will be used for further development stages (automation design and deployment). A comparison between two thermal cameras has been established from a practical point of view, in order to optimize the automation development (compactness, cost...). The two employed cameras were a cooled FLIR SC7600 based on InSb photon detector covering MWIR range and an uncooled Xenics Gobi640GigE based on microbolometer (a-Si) detector covering LWIR range. The induction system generated a maximum output power of $10 \mathrm{~kW}$ with frequency values up to $200 \mathrm{kHz}$. Visualization of the cracks is achieved with the tested parameters, and complementary tests are actually performed in order to improve detection reliability.

Image processing was performed to obtain phase images where the crack is clearly visible. The best images are obtained for the maximum induction power and large induction times (500 ms) for both cameras. Nevertheless, crack detection is clearly better with the FLIR images as expected; the FLIR camera shows a better sensitivity and temporal resolution. The coil positioning and therefore the total density of generated Eddy current have been revealed as the critical parameter for crack detection. For the Xenics camera a minimum change on the Eddy current density can avoid the crack detection.

Further analysis will be performed for evaluating the crack detection numerically from a first approach calculating the image profiles. Improving the image quality through different filtering solutions will reduce the image noise, and careful consideration for the grey gradient generated by the heating will allow most reliable profiles for this calculus. The interest of the crack detection value will help to define specific parameters value in new inspections. 


\subsection{1/qirt.2016.165}

The principal aim of this study is to develop an automated inspection system for magnetic particle substitution. The automated acquisition and image processing with the actual induction elements were demonstrated with the direct visualization of the crack in the studied pieces. Future work will involve both hardware and software development.

The hardware development will account mainly for an automated positioning and rotation for thermal visualization of the whole part. A key issue to improve defect detection will be the field of view (FOV) and piece orientation optimization. Another critical point is the optimization of the Eddy current generation; this issue is currently addressed with a specific coil design. The final system will endorse a reliable and repeatable detection.

Automated detection of the crack will be based on two principal software developments: image processing for improving general image quality and a further cognitive system for quality diagnosis. Specific processing (system training with a previous labeling stage) will be the input for a cognitive system, where regression and classification algorithms will allow the automatic crack detection by distinguishing it from other external details.

\section{Acknowledgments}

This work was supported by Xunta de Galicia, through the Ministerio de Economía e Industria, within the research project IN853A 2015/04: JOINTS 4.0.

\section{REFERENCES}

[1] Balageas D., Maldague X., Burleigh D. et al, Thermal (IR) and other NDT techniques for improved material inspection, Journal of Nondestructive Evaluation, 35(1), 1-17, 2016.

[2] Ibarra-Castanedo $\mathrm{C}$. et al, Comparative study of active thermography techniques for the nondestructive evaluation of honeycomb structures, Research in Nondestructive Evaluation, 20: 1-31, 2009.

[3] Zenzinger, G., Bamberg, J., Satzger, W., \& Carl, V., Thermographic crack detection by eddy current excitation. Nondestructive Testing and Evaluation, 22(2-3), 101-111, 2007.

[4] Netzelmann, U., Walle, G. "Induction thermography as a tool for reliable detection of surface defects in forged components", Proceedings of the $17^{\text {th }}$ World Conference on Non-destructive Testing, Shanghai (China), 2008.

[5] Oswald-Tranta, B., Thermoinductive investigations of magnetic materials for surface cracks, Quantitative InfraRed Thermography Journal, 1:1, 33-46, 2004.

[6] Vrana, J. et al, "Mechanisms and models for crack detection with induction thermography", Review of quantitative nondestructive Evaluation, Vol 21 edited by D.O. Thompson, 2008.

[7] Mook, G., Hesse, O., Uchanin, V., "Deep penetrating Eddy currents and probes", Proceedings of the $6^{\text {th }}$ European Conference on Non Destructive Testing, Berlin 2006.

[8] FLIR systems, "The ultimate infrared handbook for R\&D professionals" (www.flir.com), 2012.

[9] Redjjimi et al, "Noise equivalent temperature difference model for thermal imagers, calculation and analysis", Scientific Technical Review, 64(2), pp.42-49, 2014.

[10] Maldague, X., Introduction to NDT by active infrared thermography, Materials Evaluation Vol. 6 No. 9, pp 10601073, 2002.

[11] Franco, L. et al, "Inspection analyses on non-cured samples by lock-in and PPT thermography", $6^{\text {th }}$ Symposium for NDT in Aerospace, AeroNDT, Madrid (Spain) 2014. 